\title{
Continuidade e descontinuidade de uso de tecnologias digitais de informação e comunicação por professores universitários das Ciências e da Saúde
}

\author{
Continuity and discontinuity in using information \\ and communication technology by Health Science professors
}

\author{
Rosilaine de Fátima Wardenski ${ }^{1}$ \\ https:// orcid.org/0000-0002-3026-2971 \\ Miriam Struchiner ${ }^{1}$ \\ https://orcid.org/0000-0002-9979-2364 \\ Tais Rabetti Giannella ${ }^{1}$ \\ https://orcid.org/0000-0001-9563-2964
}

\begin{abstract}
Resumo: Com base na literatura sobre fatores envolvidos na integração das tecnnologias de informação e comunicação (TIC) no ensino, este estudo teve como objetivo analisar os aspectos que influenciam a continuidade e a descontinuidade no uso de uma ferramenta de autoria de cursos na Internet, a partir das percepções de professores de Ciências da Saúde. Realizaram-se entrevistas semiestruturadas com oito professores universitários que adotaram a ferramenta Constructore. Os resultados obtidos com a análise temática de conteúdo indicam que a continuidade/descontinuidade do uso desta ferramenta é influenciada por aspectos de diferentes naturezas: pedagógica (adesão dos alunos e reconhecimento do potencial pedagógico de TICs), pessoal (dedicação à prática docente), institucional (valorização da prática pedagógica, oferta de infraestrutura e formação) e tecnológica (simplicidade, compatibilidade entre potencialidades da ferramenta e objetivos de ensino, e estabilidade da ferramenta). Esses resultados contribuem para o conhecimento sobre a dinâmica de integração de inovações baseadas em TICs nas práticas educativas nas Ciências da Saúde.
\end{abstract}

Palavras-chave: Tecnologias de informação e comunicação. Ciências da saúde. Ambiente virtual de aprendizagem. Ensino a distância.

\begin{abstract}
Based on the scientific literature about factors related to information and communication technology (ICT) integration in teaching, this study aimed to evaluate Health Sciences professors' perceptions on the aspects that can influence continuity/discontinuity in the use of a web-based authoring tool (Constructore). We conducted semi-structured interviews with eight university professors who adopted Constructore. Responses were analyzed through the method of content analysis. Results showed perceptions regarding different aspects: pedagogical (such as student's involvement with the proposed activities and pedagogical potential of ICT in Health Science), personal (readiness to improve teaching practice), institutional (incentives for enhancement pedagogical practice, and also provision of infrastructure and training), and technological (such as simplicity, compatibility between authoring tool resources and the pedagogical goals, and also stability of the tool). These results contribute to knowledge on the integration of ICT-based innovation in Health Sciences education.
\end{abstract}

Keywords: Information and communication technologies. Health Science teaching. Virtual learning environment. Distance education.

\footnotetext{
${ }^{1}$ Universidade Federal do Rio de Janeiro (UFRJ), Núcleo de Tecnologia Educacional para a Saúde (NUTES), Rio de Janeiro, RJ, Brasil. E-mail: <rosiwardenski@gmail.com>.
} 


\section{Introdução}

Considerando as exigências de formação na área das Ciências da Saúde, diversos autores defendem o desenvolvimento de estratégias educativas inovadoras, baseadas no diálogo e na construção ativa de conhecimento pelos alunos (MCALLISTER et al., 2013; RANGEL et al., 2011; VYAS et al., 2010). Nesse contexto, as Tecnologias de Informação e Comunicação (TIC) podem apoiar o enfrentamento dos desafios desta formação, tais como o ensino predominantemente transmissivo, a fragmentação dos conteúdos, a compreensão de conceitos científicos abstratos e de difícil visualização e a complexa integração entre conhecimentos científicos e prática profissional (WEINTRAUB; HAWLITSCHEK; JOÃO, 2011).

Embora muito se discuta acerca das potencialidades das TICs para a promoção de melhorias no ensino, é necessário ressaltar que os professores devem ser encarados como protagonistas das mudanças para a construção de uma nova cultura que estimule a disseminação, a continuidade e o aprimoramento da utilização destes recursos (SILVA; MARQUES, 2011).

Promover o uso de recursos tecnológicos pelos docentes em longo prazo constitui um desafio para gestores institucionais e pesquisadores do campo da tecnologia educacional (BUABENGH-ANDOH, 2012). Como apontado na literatura, a integração de TICs no ensino é um processo gradual, já que é apenas durante a utilização que os professores têm a oportunidade de avaliar a compatibilidade de determinada tecnologia com suas necessidades cotidianas (NACHMIAS; RAM, 2009). Além disso, Mendoza, Carroll e Stern (2008) afirmam que os fatores que influenciam o professor a adotar inicialmente uma ferramenta não são os mesmos que influenciam a continuidade do seu uso. Durante a integração de TICs, o indivíduo constrói novas percepções acerca desse processo e uma sinergia entre diversos aspectos pessoais, pedagógicos, institucionais e tecnológicos influencia a continuidade/descontinuidade de uso de uma ferramenta pelo professor (CARROLL et al., 2002).

Dentre os diferentes recursos educativos baseados nas TICs, as ferramentas de autoria de cursos (Course Management System) têm sido cada vez mais utilizadas no ensino superior, tanto para a realização de cursos totalmente a distância como de atividades semipresenciais (COOK et al., 2010; GOUDOURIS; GIANELLA; STRUCHINER, 2013). Estas ferramentas oferecem aos professores a oportunidade de construir e mediar ambientes virtuais de aprendizagem (AVA), a partir do uso integrado de diferentes recursos para a disponibilização e organização da informação, comunicação/colaboração, realização de atividades e acompanhamento/avaliação da aprendizagem. Em uma revisão da literatura sobre o uso de TICs no ensino de Ciências da Saúde, Wardenski (2016) observou que há um uso crescente dessas ferramentas, tendo em vista que facilitam o trabalho do professor na construção de atividades educativas inovadoras, que aproveitem o potencial multimidiático das TICs e que possibilitem aos alunos experiências colaborativas com diferentes atores e cenários de práticas fundamentais para a sua formação.

Este artigo tem como objetivo identificar os aspectos que influenciam a continuidade/ descontinuidade no uso de uma ferramenta de autoria de cursos na Internet (Constructore) a partir das percepções de professores universitários da área das Ciências da Saúde. Assim, esta pesquisa pretende contribuir para o corpo de conhecimentos sobre a dinâmica de integração de inovações educativas baseadas em TICs, tendo em vista que, embora a literatura neste campo seja vasta, são poucos os trabalhos que têm como enfoque a análise da continuidade/descontinuidade de uso dessas ferramentas por professores universitários (WARDENSKI, 2016). 


\section{Referencial teórico}

A literatura no campo da tecnologia educacional apresenta diversos fatores que podem influenciar a decisão de professores no que diz respeito à integração de TICs no ensino (BUAHBENGH-ANDOH, 2012). Esses fatores estão relacionados a condições que facilitam ou dificultam o uso desses recursos e podem ser classificados em: pedagógicos, pessoais, institucionais e tecnológicos.

Com relação aos fatores pedagógicos, a literatura aponta diferentes potencialidades das TICs para auxiliar a formação nas Ciências da Saúde, por meio de: (i) materiais educativos em diferentes mídias para representações de conceitos e fenômenos científicos (MACALLISTER et al., 2013); (ii) ferramentas de comunicação que podem viabilizar o trabalho colaborativo em atividades, tais como análises de exames clínicos, realização de diagnósticos e estudos de caso que demandam abordagens multidisciplinares (COOK et al., 2010); e, (iii) ferramentas para a aprendizagem ativa, como portfólios reflexivos (VYAS et al., 2011) e programas de simulação que envolvem os alunos em situações realistas, de tomada de decisões e de experimentação sem os riscos da vida real (HAGGERTY, 2009; SO; BRUSH, 2008).

Enquanto Harris, Mishra e Koehler (2009) destacam que a decisão de os professores adotarem diferentes estratégias de ensino-aprendizagem potencializadas pelas TICs está relacionada com a natureza dos conteúdos de ensino, Hu e MCGrath (2012) afirmam que os objetivos de ensino podem mudar ao longo do tempo, modificando também a pertinência do uso destes recursos em suas práticas.

Outro fator pedagógico destacado na literatura é a influência do retorno dado pelos alunos, visto que a motivação e a aprendizagem dos alunos constituem o objetivo central para a implementação de melhorias no ensino (GROFF; MOUZA, 2008). Os autores afirmam que, em geral, o processo de aprendizagem mediado por tecnologias demanda grande tempo de dedicação e pode ser muito diferente das práticas experimentadas pelos alunos, exigindo maior autonomia e disciplina. Essas atividades devem ser desafiadoras para alunos acostumados com ambientes tradicionais, em que o professor é o centro do processo de aprendizagem. Os educadores devem antecipar os possíveis desafios enfrentados pelos alunos durante o desenvolvimento de projetos baseados em tecnologias digitais. Esse aspecto é importante, pois caso os alunos não demonstrem adesão às atividades propostas, os docentes não acreditam que estão alcançando seus objetivos e se sentem desencorajados a continuar o uso (MUMTAZ, 2000).

Entre os fatores pessoais, destaca-se a familiaridade com o uso das TICs. Essa familiaridade pode ser construída ao longo do desenvolvimento profissional docente, mas também se relaciona com a usabilidade da ferramenta, que facilita ou dificulta a utilização pelo professor, e influencia suas visões e atitudes sobre este processo (NIELSEN, 1999). Segundo Buahbengh-Andoh (2012), os professores com maior experiência pedagógica com estas ferramentas apresentam maior confiança em suas habilidades para utilizá-las efetivamente, aprendem mais rapidamente, além de estarem mais dispostos a usar a tecnologia para propostas educativas. Já aqueles que não se consideram habilidosos no uso de recursos tecnológicos, sentem-se ansiosos ao utilizar TICs diante de alunos com mais familiaridade no uso dessas ferramentas, o que pode levar à descontinuidade (MENDOZA; CARROLL; STERN, 2008). West, Waddoups e Graham (2007) indicam que as dificuldades com o uso de TICs nas práticas de ensino, muitas vezes, estão relacionadas aos aspectos pedagógicos da integração. Os autores afirmam, por exemplo, que 
muitos professores apresentam dificuldades em atividades de ensino online, como a moderação de discussões e acompanhamento dos alunos.

Segundo Buahbengh-Andoh (2012), a disposição dos professores para superar esses desafios está relacionada à dedicação à prática docente, que também é um fator pessoal importante para a continuidade no uso de ferramentas tecnológicas no ensino. Em geral, os professores que dedicam tempo ao planejamento, aprendizado e colaboração com seus pares apresentam maior facilidade para atingir seus objetivos educacionais com o uso de TICs (BUAHBENGH -ANDOH, 2012). Neste sentido, Mumtaz (2000) afirma que o sucesso de projetos de ensino baseados em tecnologia está relacionado a quanto o professor se sente motivado a aprimorar sua prática didática, considerando que isso afeta a motivação dos alunos. Os fatores pessoais são marcados especialmente pelas crenças dos docentes em relação ao processo educativo e ao papel das TICs. Porém, como afirmam Ertmer e Leftwich (2010), a cultura institucional em que o professor está inserido influencia sua decisão pessoal acerca da integração tecnológica.

Entre os fatores institucionais, destaca-se a necessidade de infraestrutura adequada, que inclui a disponibilidade e manutenção de ferramentas tecnológicas e acesso a rede de Internet. Quando os professores decidem integrar TICs em suas práticas educativas, eles podem se sentir frustrados e desestimulados caso os recursos utilizados apresentem problemas (CARVALHO et al., 2012). Na literatura nacional, são recorrentes os relatos de descontinuidade de uso em função de problemas como acesso à Internet (RANGEL et al., 2011). Além disso, Pelgrum (2001) afirma que a instituição deve assumir a oferta de formação para os professores. Isso ocorre porque os docentes precisam de suporte para superar os desafios técnicos e pedagógicos inerentes à integração de um novo elemento na prática de ensino. Segundo Giannella (2007), é preciso que esse suporte seja contínuo, não apenas nas questões técnicas, mas também no que diz respeito ao diálogo e troca com os professores.

Com relação aos fatores tecnológicos, os atributos de inovações definidos por Rogers (2003) podem ajudar na análise das características das TICs que influenciam as decisões dos professores sobre seu uso: (a) compatibilidade é o quanto uma inovação é percebida pelos professores como adequada às suas necessidades; (b) vantagem relativa caracteriza o quanto uma ferramenta apresenta maiores vantagens para as práticas educativas em relação a outros recursos utilizados; (c) simplicidade é a percepção do professor sobre a facilidade de uso de uma determinada TIC; (d) flexibilidade de experimentação é a oportunidade de o usuário testar e avaliar previamente a inovação; e, (e) visibilidade é o grau com que os benefícios da inovação são visíveis à comunidade educacional. Diferentes estudos analisam a influência destes atributos tecnológicos na adoção inicial de uma determinada tecnologia (BUAHBENGH-ANDOH, 2012). A presente análise tem como foco sua influência na continuidade/descontinuidade de uso.

\section{Metodologia}

Este trabalho foi desenvolvido no contexto de uso da Constructore, uma ferramenta de autoria de cursos na Internet, construída com o objetivo de apoiar professores universitários de Ciências da Saúde a integrar as TICs, promovendo melhorias no ensino e superando os desafios inerentes a esse processo (GIANNELLA, 2007). Essa ferramenta foi idealizada e desenvolvida em parceria com professores da área das Ciências da Saúde, sendo remodelada 
a partir das necessidades desses usuários. A pesquisa sobre o uso da Constructore vem se consolidando como um espaço oportuno de colaboração, experimentação e análise em que princípios e pressupostos educacionais são modelados na ferramenta e esta se configura como lente para investigar as diferentes formas de utilização das TICs no ensino das Ciências da Saúde (GOUDOURIS; GIANNELLA; STRUCHINER, 2013). Aspectos de sua usabilidade foram avaliados em estudos anteriores (GIANNELLA, 2007; RAMOS, 2004).

A Constructore ${ }^{2}$ apresenta uma página inicial e três ambientes internos principais: (1) ambiente do professor - primeira página à qual o professor tem acesso, após se cadastrar e fazer o login na ferramenta; (2) ambiente de criação do curso - onde o professor inicia a construção de seu AVA, seguindo diversas etapas de criação; e, (3) o ambiente do curso - este último é constituído pelas seguintes áreas: (a) "página inicial" - apresentação do curso e informações sobre o público-alvo, orientações gerais aos alunos, ementa e cronograma; (b) "módulos" - compostos por uma página inicial com uma apresentação de seus objetivos e atividades, pelo espaço dos objetos de aprendizagem onde o professor insere e organiza os materiais, pelo espaço das atividades e por um espaço de elaboração de formulários; (c) "comunicação" - com os recursos de avisos, fórum, e-mail e perguntas frequentes; (d) "consulta" - que reúne os recursos de glossário, links e bibliografia; (e) "participantes", com a lista de professores, tutores, monitores e alunos inscritos; e, (f) "gerência" - com recursos para acompanhamento do curso.

Entre os anos de 2007 a 2013, período de realização deste estudo, a Constructore foi utilizada na Universidade Federal do Rio de Janeiro (UFRJ) por 52 professores envolvidos com a oferta de cursos voltados para alunos das Ciências da Saúde. Durante esse período, o conjunto de docentes ofereceu 103 cursos para 509 alunos com a Constructore: 59 cursos de graduação, 28 de pós-graduação e 16 de extensão.

Tendo como objetivo auxiliar a seleção dos sujeitos da pesquisa, os professores usuários da Constructore foram classificados em quatro perfis de acordo com a frequência/continuidade de uso: Uso continuado $(\mathrm{n}=6)$ : utilizaram a Constructore pelo menos uma vez ao ano, desde que começaram; Uso intermitente $(n=18)$ : utilizaram a ferramenta duas ou mais vezes, com intervalos de dois ou mais semestres sem utilizá-la; Uso continuado seguido de desuso $(n=4)$ : apresentaram uso continuado como descrito na primeira categoria, seguido de quatro ou mais semestres sem utilizá-la e Uso pontual ( $n=24)$ : utilizaram a ferramenta apenas uma vez.

Para a realização do estudo foram sorteados e convidados quatro professores pertencentes a cada perfil de uso. Como pode ser observado no quadro 1, oito professores aceitaram o convite e participaram de entrevistas semiestruturadas, que tiveram como objetivo identificar suas percepções sobre os aspectos que influenciaram suas decisões em continuar (ou não) o uso da Constructore em suas atividades de ensino.

As entrevistas foram transcritas e analisadas com base na análise temática de conteúdo de Bardin (2004), buscando identificar, de acordo com o referencial apresentado, aspectos pedagógicos, pessoais, institucionais e tecnológicos envolvidos em suas experiências de uso com a ferramenta.

\footnotetext{
2 Disponível em: <http://ltc.nutes.ufrj.br/constructore>. Acesso em: 14 ago. 2018.
} 
Quadro 1. Relação de disciplinas oferecidas com a Constructore pelos professores participantes do estudo

\begin{tabular}{|c|c|c|}
\hline $\begin{array}{l}\text { Frequência de } \\
\text { uso }\end{array}$ & Professor & $\begin{array}{l}\text { Disciplinas em que a } \\
\text { Constructore foi utilizada }\end{array}$ \\
\hline \multirow[t]{3}{*}{$\begin{array}{l}\text { Uso } \\
\text { continuado }\end{array}$} & P1 & $\begin{array}{l}\text { Planejamento curricular e de ensino na área da Saúde (pós-graduação) } \\
\text { Formação pedagógica de preceptores do ensino em Saúde (extensão) }\end{array}$ \\
\hline & P2 & $\begin{array}{l}\text { Bioquímica (graduação) } \\
\text { Adaptações metabólicas (pós-graduação) } \\
\text { Hormônios relacionados ao controle de peso corporal (extensão) }\end{array}$ \\
\hline & P3 & $\begin{array}{l}\text { Aprendendo a aprender na área da Saúde (graduação) } \\
\text { Metodologia científica (pós-graduação) }\end{array}$ \\
\hline \multirow{3}{*}{$\begin{array}{l}\text { Uso } \\
\text { continuado } \\
\text { seguido de } \\
\text { desuso }\end{array}$} & P4 & $\begin{array}{l}\text { Boas práticas de laboratório (graduação) } \\
\text { Fisiologia renal (graduação) }\end{array}$ \\
\hline & P5 & Estrutura e função de proteínas (graduação) \\
\hline & P6 & Neurofisiologia (graduação) \\
\hline \multirow[t]{2}{*}{ Usopontual } & P7 & Bioquímica para Odontologia (graduação) \\
\hline & P8 & Concepções teóricas de Enfermagem (graduação) \\
\hline
\end{tabular}

Fonte: Elaborado pelos autores.

\section{Resultados e discussão}

\section{Aspectos pedagógicos}

Todos os sujeitos apontaram que a Constructore possui recursos que vão ao encontro de seus objetivos pedagógicos e favorecem o ensino das Ciências da Saúde.

A maioria dos entrevistados (P1, P2, P4, P5, P6, P7 e P8) ressaltou a importância da aquisição de conceitos científicos pelos alunos e destacou o papel das tecnologias como importante ferramenta para a organização e disponibilização de diversos tipos de materiais, além de ajudar a direcionar o estudo, já que os alunos possuem acesso às informações previamente selecionadas e organizadas pelo professor. "Eu acho que esse é o papel de você ter uma ferramenta, porque serve também como um instrutor do que estudar ou que materiais realmente utilizarpara aquele estudo." [P6 - uso continuado seguido de desuso].

Pensando nas necessidades específicas das Biociências ou da área da saúde, alguns professores destacaram a possibilidade de disponibilizar materiais que facilitem a visualização de estruturas e fenômenos abstratos. Nesses casos, o professor pode inserir conteúdos no próprio AVA (P2, P4 e P7) ou indicar links para outras ferramentas, como softwares e programas de computador (P5 e P6). "As neurociências, ou o que eu ensino, de anatomia, fisiologia etc., usa muito recurso audiovisual. Epra isso as tecnologias são ferramentas ótimas." [P6 - uso continuado seguido de desuso].

A maioria dos sujeitos destacou, também, a necessidade de promover a formação crítica do profissional da área da saúde (P1, P3, P5, P6 e P8), visto que os alunos têm que lidar com diversas informações durante a vida profissional, visando contemplar as ações de prevenção, promoção e reabilitação da saúde. Ressaltaram que o aluno precisa refletir e integrar seus conhecimentos a fim de tomar decisões mais adequadas para cada indivíduo atendido.

Os docentes propuseram, por meio de seus AVAs, atividades que estimulam os alunos a reunir diferentes informações para que possam encontrar a solução de algum problema 
previamente dado (P1, P2, P3, P7 e P8). A professora P1, por exemplo, relatou a aplicação da metodologia da aprendizagem baseada em casos, que tem como objetivo trabalhar os conceitos de forma contextualizada com o cotidiano do trabalho em saúde, dessa forma estimulando os alunos a pesquisar, discutir e resolver problemas relacionados à prática clínica.

A gente tenta trazer as coisas que o médico vive no dia a dia, quando ele está interagindo com o paciente e com o aluno. O aluno comesa lendo o problema, pode tirar dúvidas, ver se tem alguma coisa que ele não entendeu [...] Sistematizar as informações, pode ver o que tem de importante, discutir entre eles, pesquisar materiais que possam ajudar a resolver, e tentar chegar a uma conclusão final. [P1 - uso continuado].

Como apoio à realização das atividades, esses professores apontaram a utilização do fórum de discussão para promover a colaboração entre os alunos e estimular o compartilhamento de informações e experiências.

Eu queria que fosse um aluno mais reflexivo e acho que um fórum ajudaria nisso. Por isso que uma plataforma de ensino a distancia é tão interessante. O cara tem que raciocinar, dar opinião, e ele não costuma fazer isso em uma sala de aula em que ele fica sentado enquanto a professora fala. [P7 - uso pontual].

Diferentemente de grande parte dos relatos da literatura que indica que os professores utilizam as TICs somente para a oferta de conteúdos (COOK et al., 2010; GOUDOURIS, GIANNELLA; STRUCHINER, 2013), a maioria dos docentes entrevistados procurou integrar os AVA à dinâmica das disciplinas, demonstrando a necessidade de fazer com que o uso atendesse diferentes preocupações que sentiam em relação ao ensino das Ciências da Saúde. A intenção de promover mudanças no processo educativo pareceu um aspecto importante para que esses docentes utilizassem a Constructore. No entanto, como discutem Hu e MCGrath (2012), ao longo do tempo, não apenas os objetivos do professor podem mudar como a interferência de outros fatores pode influenciar a continuidade de uso. Embora os professores entrevistados tenham afirmado que as tecnologias podem apoiar a formação na área da saúde, a adesão dos alunos é essencial para o êxito das atividades planejadas e desenvolvidas.

Neste sentido, os professores P4, P6, P7 e P8 relataram que a baixa adesão dos alunos os desestimulou a continuar integrando os AVAs em suas disciplinas:

O que eu percebi nos estudantes, e isso te desestimula a usar as ferramentas, é que ainda bá uma cultura muito grande do presencial [...] O que eu percebi é que eles não viram a plataforma como um ambiente de aprendizagem, no sentido mais abrangente [...] do compartilhamento, de uma atividade intensa com o uso da ferramenta. É muita coisa desse tipo. Oh! Beleza, o material está aqui, é aqui que eu vou receber a informação do professor. Eles não enxergaram que aquilo ali poderia ter dado um pulo do gato muito maior pra eles. [P8 - uso pontual].

Daí você não vê o pessoal acessando isso como um recurso real. E aí você acaba pensando o pró e o contra, né, de usar? O peso, o quanto trabalho você tem e o retorno que ta 
tendo, né? E principalmente também é isso, a questão do mau aproveitamento por parte dos alunos. [P6 - uso continuado seguido de desuso].

Os docentes apontaram que esse quadro poderia estar relacionado ao baixo interesse dos alunos pelo estudo (P4, P6, P7 e P8). A professora P4, por exemplo, destacou que os alunos apresentam maior interesse pelas disciplinas específicas da sua formação em detrimento das ciências básicas, não valorizando, muitas vezes, a aprendizagem de Neurofisiologia na Educação Física. "Quer dižer, os alunos não se interessam pelo que está aqui porque eles acham que não vão utilizar pra nada [...] eles não conseguem visualizar que isso é importante pra eles." [P4 - uso continuado seguido de desuso].

$\mathrm{Na}$ percepção de $\mathrm{P} 4, \mathrm{P} 6$ e $\mathrm{P} 7$, os alunos quase nunca acessavam os AVA devido à não obrigatoriedade de seu uso. Para eles, isso estava relacionado à falta de iniciativa desses alunos para aprender conteúdos de determinada disciplina quando não havia cobrança direta. Ressaltase que a utilização pelos alunos é amplamente influenciada pela maneira como os professores integraram a Constructore na disciplina, ou seja, se os recursos da ferramenta foram ou não utilizados como parte de uma proposta pedagógica bem consolidada (MENEGHEL; KREISCH, 2009). Por isso, esses aspectos são discutidos posteriormente, como parte das características pessoais dos professores. "O problema foi a minha estratégia, porque en deixei livre. O acesso era livre, e eles acham que não podia ser livre, que tinha que valer ponto. Caramba, sabe? Os alunos estão na faculdade e eles precisam que a gente cobre isso deles?' [P7 - uso pontual].

Essas percepções dos professores sobre a atitude dos alunos em relação ao processo de aprendizagem com TICs vão ao encontro do que é relatado na literatura. Como apontam Coutinho e Lisbôa (2011), ainda não há uma cultura de aprendizagem online estabelecida e, embora os jovens usem as TICs cotidianamente, ainda não as valorizam como ferramentas de aprendizagem. Por outro lado, diversos autores apontam que dificilmente os alunos aderem a metodologias de ensino baseadas nas TICs quando percebem que seu uso não é priorizado pelos docentes (MCALLISTER et al., 2013). Além disso, o modo como os alunos estudam está relacionado às tradicionais formas de avaliação utilizadas pelos professores que, muitas vezes, não contemplam uma análise continuada do processo de aprendizagem (MENEGHEL; KREISCH, 2009).

Como aponta Buahbengh-Andoh (2012), as atitudes de professores e alunos se influenciam e uma boa interação entre eles é fundamental para o sucesso de projetos educativos baseados em TICs. Assim, se os alunos não utilizam estes recursos, os professores tendem a descontinuar seu uso, pois não conseguem atingir seus objetivos educacionais. No entanto, essa relação é permeada, também, por outros aspectos discutidos a seguir.

\section{Aspectos tecnológicos}

Com relação à compatibilidade (ROGERS, 2003), como indicaram os resultados relacionados aos aspectos pedagógicos, todos os professores apontaram encontrar na Constructore recursos apropriados a seus objetivos de ensino, já que promovia maior aproximação com os alunos por meio dos recursos de comunicação e de disponibilização, compartilhamento e organização de materiais e informações.

Porém, como discute Buahbengh-Andoh (2012), a compatibilidade não é uma característica intrínseca da inovação e pode mudar ao longo do tempo, incluindo uma série de aspectos 
relacionados ao perfil pessoal e profissional do professor, como as competências pedagógicas e tecnológicas para o desenvolvimento de atividades baseadas em TICs, e as intenções quanto ao processo educativo.

A professora $\mathrm{P} 4$, por exemplo, demonstrou incertezas com relação a essa compatibilidade, à medida que foi utilizando seu AVA. Ela afirmou que seu objetivo com o uso da ferramenta foi oferecer um repositório de recursos audiovisuais para auxiliar os alunos na aprendizagem de conceitos e fenômenos fisiológicos. Em princípio, a ferramenta foi considerada compatível, mas como ela foi desenvolvida a fim de oferecer condições para a oferta de cursos, acabava por lhe demandar mais tempo de trabalho. Assim, ela preferiu utilizar a ferramenta de e-mail, que era mais simples.

Então, durante todo o tempo que eu usei a Constructore, en usei como repositório de arquivo basicamente e meio de comunicação com os alunos. [...]. E foi por isso que eu parei de usar, porque no final das contas era muito trabalhoso. Eu abria, os alunos se inscreviam, eu liberava. Hoje em dia é mais fácil, eu mando os slides pro e-mail da turma. [P4 - uso continuado seguido de desuso].

Este exemplo nos mostra que a percepção do professor acerca da compatibilidade de uma ferramenta não está ligada apenas aos aspectos tecnológicos, mas é influenciada por diversos fatores, incluindo as características pessoais dos professores, como apontado por Giannella (2007). Por isso, consideramos importante destacar a visão que o sujeito apresenta acerca do processo de ensino-aprendizagem, que, no caso de P4, não inclui processos colaborativos. Essa professora afirmou ter apresentado dificuldades para compreender como utilizar a Constructore de maneira mais proveitosa para si e para os alunos, e isso está mais bem discutido posteriormente, em aspectos pessoais.

Com relação à facilidade/complexidade de uso, todos os professores apontaram que a Constructore é uma ferramenta de fácil utilização, e serviu ainda como um aprendizado inicial para a utilização pedagógica de TICs. A professora $\mathrm{P} 7$, por exemplo, apontou que, devido à imposição institucional, precisou usar outra ferramenta tecnológica de autoria de cursos na internet, o Moodle ${ }^{3}$, de uso livre. Segundo ela, embora tenha considerado essa ferramenta mais difícil de utilizar, a experiência vivida anteriormente facilitou sua adoção. Confirmando isso, Mendoza, Carroll e Stern (2008) apontam que a dificuldade é um aspecto que perde importância ao longo do tempo, já que o sujeito ganha confiança para manejar a tecnologia.

Um fator que dificultou a utilização da ferramenta pela maior parte dos sujeitos (P1, P2, P3, P4, P5, P6, e P7) foi a presença de falhas técnicas, especialmente com relação à estabilidade do sistema, ocasionadas por queda de energia. Um dos professores afirmou que estava pensando em descontinuar o uso em virtude deste aspecto: "Há formas e sugestôes para a Constructore melhorar? Sim. Mas não é isso que me faria abandonar a Constructore. O que me faria abandonar é a estabilidade." [P3 - uso continuado].

\footnotetext{
${ }^{3}$ Disponível em: <https://moodle.org/?lang=pt_br>. Acesso em: 3 mar. 2015.
} 
Entretanto, a maior parte dos sujeitos (P1, P2, P4, P5 e P6) afirmou que esses problemas não os impediram de continuar utilizando a ferramenta. Ressalta-se que a Constructore foi desenvolvida em um processo de parceria com os usuários, sendo continuamente remodelada para atender às suas solicitações (GIANNELLA, 2007). Nesse contexto, a maior parte dos entrevistados recebeu acompanhamento dos pesquisadores do Laboratório de Tecnologias Cognitivas ao longo do uso, o que, segundo os professores, foi fundamental para sua integração (P1, P2, P3, P5 e P6).

Como já ressaltado, para perceber vantagens e compatibilidade nos recursos presentes em determinada ferramenta, os docentes precisam aprender a manuseá-la e se familiarizar com seu uso (WEST; WADDOUPS; GRAHAM, 2007). Existe uma tendência a considerar os desafios técnicos como secundários, em relação aos pedagógicos. Mas é possível que estes sejam uma das principais barreiras e fontes de insegurança para os professores, que precisam vivenciar um processo de experimentação e internalização dos recursos e do funcionamento, para posteriormente focar nas possibilidades pedagógicas (PELGRUM, 2001). Por outro lado, destaca-se que o desenvolvimento de conhecimento sobre os aspectos tecnológicos deve ser articulado à dimensão pedagógica de integração das TICs, afastando-se de grande parte dos modelos de formação de professores, que ainda perpetua uma visão instrumental deste processo (GOUDOURIS; GIANNELLA; STRUCHINER, 2013).

\section{Aspectos pessoais}

Considerando que a integração de TICs no ensino de forma proveitosa demanda tempo e disponibilidade (BUAHBENGH-ANDOH, 2012), os professores que apresentaram maior envolvimento com o planejamento e desenvolvimento das atividades oferecidas por meio da Constructore, de forma geral, foram aqueles que continuaram utilizando a ferramenta por maior período (P1, P2 e P3). Esses sujeitos foram aqueles que apontaram ter percebido maior participação dos alunos, e assim, tiveram maior êxito em atingir seus objetivos educacionais com o uso de TICs, percebendo mudanças qualitativas no processo de ensino-aprendizagem. É neste sentido que os aspectos pessoais demarcam as visões e atitudes desenvolvidas pelos docentes sobre a integração de TICs ao longo do tempo.

Assim, os professores P1, P2 e P3 relataram que utilizaram a Constructore para aprimorar estratégias de ensino-aprendizagem que já vinham planejando há certo tempo. Desta maneira, estes professores reforçaram que, ao integrar a ferramenta, precisaram repensar a dinâmica de ensino, de maneira a aproveitar o potencial de atividades presenciais e à distância.

Na verdade eu não tive muitas dificuldades [...]. Porque en trabalbo muito com a aprendizagem baseada em problemas, [...] e o que a gente tenta fazer com isso é trazer alguns problemas que o médico vive no dia a dia [...]. Eu já faz̨ia isso, já utilizava essa estratégia, mas a Constructore me ajudou muito, para facilitar a discussão, ter mais um meio para os alunos entrarem em contato, como com o fórum de discussão. Deu para inserir alguns materiais também para servirem de base. E como a ferramenta é bem fácil de usar, eu sóprecisei adaptar as coisas para o ambiente. [P1 - uso continuado]. 
Estes professores visualizaram na Constructore recursos que os ajudariam a alcançar objetivos de ensino que já estavam delineados, e por isso, sua tentativa de promover melhorias no processo de ensino-aprendizagem obteve maior sucesso. Consequentemente, esses professores buscaram mais alternativas para superar os desafios e continuar o uso da ferramenta. A professora P1, por exemplo, indicou que teve a oportunidade de aprender a mediar discussões online por meio da prática com o uso do fórum; enquanto P2 mencionou algumas reclamações de alunos com relação ao uso da Constructore, destacando que precisou explicar claramente que tipo de atividade seria realizada por meio da ferramenta.

Muitas vezes eles pensam que é só pra pegar os slides, porque já devem ter tido outra experiência com um professor que usava pra disponibilizar as aulas. Aí eu falo: não! Prestem atenção que não é isso que vocês vão fažer, é realmente uma atividade. [P2 uso continuado].

Os professores P7 e P8, classificados na categoria de uso pontual, demonstraram a intenção de implementar atividades de ensino dinâmicas, que utilizassem amplamente os recursos encontrados na Constructore. Entretanto, eles reconheceram que não planejaram a forma como a ferramenta seria integrada na disciplina, afirmando que isso pode ter contribuído para o desuso.

A disciplina não foi adaptada. Ai agora que você me fez essa pergunta, eu penso que talvez ela tivesse que ter sido adaptada, que talvez tivesse que ter sido reconstruida. Não sei até que medida isso não foi feito, e eu tenha falhado nisso. [P8 - uso pontual].

Ressalta-se que esses professores estavam entre aqueles que apontaram baixa adesão dos alunos às atividades propostas com a Constructore. Eles relataram ter disponibilizado materiais e fóruns de discussão para que os alunos pudessem consultar e tirar dúvidas, mas que não houve a participação esperada.

P7, por exemplo, no momento da entrevista, tinha desistido de utilizar a Constructore em uma disciplina do primeiro período de Odontologia devido à baixa adesão dos alunos. No entanto, apontou que ao planejar um novo curso na Constructore (de extensão), percebeu que na experiência com a graduação não tinha conseguido integrar a ferramenta plenamente, o que pode ter influenciado a atitude dos alunos. "[...] A gente tem que adaptar a disciplina para integrar a ferramenta. Eu tava conversando isso com os outros professores, como é que a gente vai adaptar para o próximo semestre." [P7 - uso pontual].

Essa professora passou por um período de familiarização com a ferramenta, o que pode ter servido como uma possibilidade de aprendizagem prática. Isso facilitou a construção de um novo curso, na medida em que ela pôde avaliar quais as principais lacunas do primeiro. Segundo West, Waddoups e Graham (2007), ao adotarem novos recursos, os docentes comumente tendem a usá-los para reproduzir o que já fazem, de maneira mais rápida e fácil. Os autores denominam esse comportamento como experimentação, uma vez que ao longo do tempo novos materiais podem ser incorporados à prática pedagógica. Destacam que essa é uma característica natural do processo de integração de TICs e que precisa ser levada em consideração em experiências de formação docente. 
Já os professores P4, P5 e P6, que descontinuaram o uso, indicaram que seus AVA tinham como objetivo principal oferecer recursos de conteúdo para os alunos. Justificando sua forma de utilizar o AVA, P4 apontou dificuldades a incorporar novas potencialidades pedagógicas: "Eu usei apenas como repositório de arquivo. Eu não consegui visualizar, talvez por resistência minha, outras coisas, ferramentas que a Constructore poderia me dar." [P4 - uso continuado seguido de desuso].

É possível que o uso restrito do AVA como repositório de materiais reflita a pequena motivação destes professores com o aprimoramento de suas práticas de ensino e que isso tenha influenciado, também, a descontinuidade do uso, cenário compatível ao que é relatado na literatura (COOK et al., 2010; MCALLISTER et al., 2013).

Vale destacar que, para P5, um outro fator pessoal que o levou a descontinuar o uso da Constructore, foi o fato de deixar de oferecer a disciplina Estrutura e Função de Proteínas no curso de Medicina. Segundo o professor, a principal motivação para o uso do AVA era que o período de duração da disciplina era muito curto e precisava contar com atividades semipresenciais. Ao passar a oferecer a disciplina para outro curso de maior duração (Nanotecnologia) não via mais a necessidade de integrar o AVA, posto que lhe conferia mais trabalho.

Eu não uso agora mais, porque eu tenho mais tempo com os alunos. Então aquela atividade que en fazia de forma concentrada, eu to fazendo de forma mais diluida ao longo do periodo, então eu não senti tanta necessidade de usar a Constructore [...] e isso me dava um bom trabalho. [P5 - uso continuado seguido de desuso].

Este quadro pode estar relacionado às dificuldades e inseguranças dos indivíduos no processo de integração de TICs, já que, como apontam Gonçalves e Oliveira (2011), propostas de mudança geram ansiedade por parte dos sujeitos, além de sobrecarga de trabalho. Entretanto, deve-se considerar que há, também, muitos fatores externos influenciando as práticas docentes. A adaptação de uma disciplina para a modalidade semipresencial, juntamente com o planejamento e a implementação de estratégias didáticas inovadoras, demandam apoio institucional, como infraestrutura adequada e formação docente (GROFF; MOUZA, 2008). Além disso, este processo exige tempo de dedicação do professor, como apontado por alguns professores ao longo deste estudo (P3, P4 e P8).

\section{Aspectos institucionais}

Em relação ao papel institucional, alguns professores demonstraram perceber um clima de aparente incentivo por parte da Universidade para integrarem TICs. No entanto, destacaram a precariedade das condições e do apoio necessário para que esse processo ocorresse, tanto em termos de valorização das práticas docentes que incentivassem o desenvolvimento de inovações pedagógicas, quanto em termos de oferta de infraestrutura tecnológica (P3, P4 e P6). "O que eu vejo é uma posição bivalente. Do ponto de vista do discurso oficial, estamos no século XXI e precisamos usar as TICs [...]. Do ponto de vista da ação concreta, é uma instituição em que o modelo de aula é cuspe e giz:" [P7 - uso pontual].

Neste sentido, alguns professores (P2, P4, P5 e P6) apontaram que praticamente não encontram espaço para dialogar e compartilhar informações acerca das metodologias de ensino e, consequentemente, das formas de integração de recursos tecnológicos. Assim, indicaram não 
contar com o respaldo nem com exemplos de integração de tecnologias por outros professores, o que segundo Rogers (2003), é um elemento significativo durante o processo de difusão de inovações. Isso ocorre porque a visibilidade de uma inovação (seu alcance de difusão) potencializa sua adoção, por aumentar a probabilidade dos usuários a conhecerem. (ROGERS, 2003).

Alguns professores destacaram a grande cobrança pela produção acadêmica, além de ocupações relacionadas com a administração e gestão universitária (P3, P4 e P8). O professor P8, por exemplo, relatou as inúmeras atribuições do cotidiano docente como motivos para sua decisão de descontinuar o uso da Constructore, já que o acompanhamento de um AVA demanda alta carga de trabalho. A vivência deste docente constitui um exemplo em que as dificuldades encontradas para a implementação das atividades planejadas por meio do AVA acabaram por superar as possíveis potencialidades de sua integração.

Eu fazia isso concomitantemente ao fato de ser coordenador de curso de graduação e professor de todo o resto [...]. O problema é o seguinte: com a minha ideia de criar dois ambientes sinergéticos, é como se eu tivesse que dar aula em dois lugares diferentes. Então teria discussão na sala de aula, e discussão no fórum, aí duplicava o processo. [P8 - uso pontual].

Para Cunha (2006), o prestígio do professor universitário alicerça-se, basicamente, nas atividades de pesquisa e nas orientações de dissertações e teses, além dos cargos de administração. Simões (2004) destaca que esse quadro influencia o surgimento de uma cultura da performatividade, em que os docentes apresentam uma preocupação crescente em realizar maior número de publicações. $\mathrm{O}$ autor ressalta que esta lógica traz consequências para o ensino, tendo em vista que o planejamento didático, na maior parte das vezes, é negligenciado.

Outro ponto apontado por alguns professores (P1, P2, P3, P4e P8) foi a falta de investimento e manutenção de ferramentas tecnológicas. Segundo eles, como as tecnologias utilizadas apresentavam problemas de funcionamento, eles perdiam muito tempo para concluir tarefas e atividades. "Eu acho que a estrutura nesse ponto é muito falha. A gente não tem uma rede, e quando tem uma rede boa é com senhas que você não tem acesso." [P4 - uso continuado seguido de desuso].

$\mathrm{Na}$ literatura, muitos autores citam a necessidade de disponibilizar equipamentos adequados nas instituições de ensino que passam por processos de integração de TICs, além de prover alternativas para o caso de ocorrer problemas com as ferramentas (BUAHBENGH -ANDOH, 2012; MUMTAZ, 2000).

O estímulo à inserção de TICs pela instituição de ensino também inclui a formação oferecida aos professores para o manejo e a prática pedagógica com seu uso. Diversos sujeitos afirmaram que conheceram a Constructore por meio do contato com os pesquisadores responsáveis pelo desenvolvimento da ferramenta (P1, P2, P3 e P6) e tiveram a oportunidade de receber treinamento e acompanhamento.

Então eu acho que capacitação é chave. Ou então você bota um técnico só pra inserir os dados pros professores, mas dai eu acho que perde um pouco a questão, o professor não seria mais o usuário da ferramenta, ele não faria o diálogo direto com a ferramenta. [P6 - uso continuado seguido de desuso]. 
Já P4, P5, P7 e P8 conheceram a Constructore por meio de colegas de trabalho, que indicaram sua utilização para promover um apoio às aulas presenciais. Ressalta-se que esses sujeitos não receberam acompanhamento quando começaram a utilizá-la, o que segundo P5 e P8, gerou dificuldade no manejo da ferramenta e na percepção de como utilizar seus recursos de forma efetiva no ensino. "De repente seria legal uma oficina de divulgação pra tentar entender e usar todos os recursos. [...]. Então, eu acho que de repente faltou mais conhecimento do que eu poderia alcançar com a Constructore." [P4- uso continuado seguido de desuso].

A literatura aponta que a formação para o uso de TICs constitui um aspecto que pode aumentar a confiança para a integração de ferramentas tecnológicas (BUAHBENGH-ANDOH, 2012). De forma semelhante, analisando a difusão de inovações tecnológicas em diversos contextos, Pelgrum (2001) constatou que em instituições que proviam auxílio técnico para os docentes utilizarem as TICs, a falta de habilidades técnicas não foi apontada como um fator que dificultava a integração tecnológica.

\section{Considerações finais}

Tendo como referencial estudos sobre integração de TICs ao longo do tempo (MENDOZA, 2008; WEST; WADDOUPS; GRAHAM, 2007), esta pesquisa teve por objetivo identificar e analisar as percepções de professores universitários das Ciências da Saúde sobre aspectos relevantes que contribuiram para suas decisões de continuarem ou descontinuarem o uso de uma ferramenta de autoria de cursos na Internet em suas práticas educativas.

A análise das entrevistas com os professores envolvidos identificou uma série de aspectos que foram agrupados, de acordo com suas características, em pedagógicos, tecnológicos, pessoais e institucionais. Estes aspectos são interdependentes e, muitas vezes, difíceis de analisar separadamente. Neste sentido, reforçando a complexidade do processo de integração de TICs, destaca-se a dificuldade encontrada pelos professores em definir esses aspectos nas entrevistas, especialmente por aqueles que continuaram utilizando a ferramenta.

Assim, neste trabalho, foi possível identificar com maior especificidade os aspectos que influenciaram a descontinuidade: a pequena motivação para dedicação à prática docente com TICs (aspecto pessoal - P4, P5 e P8) e a baixa adesão dos alunos às atividades propostas com os AVA (aspecto pedagógico - P6 e P7). A pequena motivação dos professores e a baixa adesão dos alunos resultam de problemas tecnológicos e de infraestrutura, assim como também de questões culturais, da proposta pedagógica e do modo de uso das TICs (BUAHBENGH -ANDOH, 2012).

Estes foram os aspectos mais marcantes nas falas dos professores, embora muitas vezes estivessem relacionados a aspectos de outras naturezas. Assim, por exemplo, a baixa adesão dos alunos aos cursos de P6 e P7 pode ter sido influenciada pela forma com que estes professores utilizaram o AVA, não tendo a preocupação em integrar atividades presenciais e à distância. Esta é uma relação bastante relatada na literatura que indica que o uso de AVA no ensino de Ciências da Saúde ainda tem um papel de apoio à transmissão de conteúdos científicos, mais do que espaço coletivo de construção de conhecimento (OWSTON, 2007). Além disso, é muito comum haver uma incompatibilidade de expectativas e interesses em relação ao uso de TICs, entre professores e alunos, não só no que diz respeito aos seus objetivos de ensino-aprendi- 
zagem, mas às potencialidades dessas ferramentas (GIANNELLA, 2007; MORAIS; ALVES; MIRANDA, 2013).

As percepções de P8 também ilustram a convergência de aspectos, neste caso, pessoais e institucionais. Se por um lado, o professor assume não ter se dedicado suficientemente à integração do AVA à sua disciplina; por outro, relata a pequena valorização da prática pedagógica em relação às atividades de pesquisa, por parte das instituições de ensino. Esse aspecto tem dificultado o desenvolvimento e o sucesso de experiências inovadoras com TICs no ensino superior (MORAIS; ALVES; MIRANDA, 2013).

Embora não tenha sido possível identificar especificamente os aspectos que influenciaram a continuidade no uso da Constructore, dada a sinergia entre eles, pode-se afirmar que todos os professores destacaram suas potencialidades pedagógicas para o ensino das Ciências da Saúde. Como discutem diversos autores (NACHMIAS; RAM, 2009), há uma crescente preocupação na construção de currículos inovadores que colocam os alunos no centro do processo educativo, preparando-os para acompanhar criticamente os avanços nas Ciências da Saúde e envolvendo-os em processos de investigação científica. As TICs têm sido importantes aliadas neste processo de transformação, que é lento e demanda tempo, experimentação e reflexão por parte dos professores (CARROLL et al., 2002).

Desta maneira, ressalta-se a contribuição de estudos como este, que se dedicam a compreender a continuidade do uso de TICs ao longo do tempo. Isto porque, diferentemente das primeiras atividades em que os professores tendem a replicar suas práticas tradicionais, é com o uso continuado que os docentes ganham experiência e refletem sobre ela e, portanto, têm a oportunidade de construir iniciativas inovadoras de ensino-aprendizagem (OWSTON, 2007).

Finalmente, destaca-se a importância de se fomentar oportunidades de formação/ aprendizagem docente em serviço para que o processo de integração de TICs seja construído de maneira situada nos problemas de ensino das Ciências da Saúde, a partir das preocupações e vivências daqueles que podem, de fato, promover melhorias na formação científica e cidadã dos futuros profissionais desta área.

\section{Agradecimentos}

As autoras agradecem o apoio do Conselho Nacional de Desenvolvimento Científico e Tecnológico (CNPq).

\section{Referências}

BARDIN, L. Análise de conteúdo. Lisboa: Edições 70, 2004.

BUAHBENGH-ANDOH, C. Factors influencing teachers' adoption and integration of information and communication technology into teaching: a review of the literature.

International Journal of Education and Development using Information and Communication Technology, Bridgetown, v. 8, n. 1, p. 136-155, 2012. 
CARVALHO, R. et al. Tecnologias de informação e comunicação e educação a distância na formação em saúde: uma revisão da literatura. In: ENCONTRO NACIONAL DE ENSINO DE CIÊNCIAS DA SAÚDE E DO AMBIENTE, ENECiências, 3., 2012, Niterói. Anais... Disponível em: < https://tinyurl.com/y6unoy2l>. Acesso em: 14 ago. 2018.

CARROLL, J. et al. Just what do the youth of today want?: technology appropriation by young people. In: ANNUAL HAWAII INTERNATIONAL CONFERENCE ON SYSTEM SCIENCES, $35^{\text {th }}$, 2002, Big Island, Hawaii. Proceedings... Los Amitos: IEEE, 2002. p. 131.2.

COOK, D. A. et al. What do we mean by web-based learning?: a systematic review of the variability of interventions. Medical Education, Oxford, v. 44, n. 8, p. 765-774, 2010.

COUTINHO, C. P.; LISBÔA, E. S. Sociedade da informação, do conhecimento e da aprendizagem: desafios para a educação no século XXI. Revista de Educação, Lisboa, v. 18, n. 1, p. 5-22, 2011.

CUNHA, M. I. Docência na universidade, cultura e avaliação institucional: saberes silenciados em questão. Revista Brasileira de Educação, Rio de Janeiro, v. 11, n. 32, p. 258-271, 2006.

ERTMER, P.; LEFTWICH, O. Teacher technology change: how knowledge, beliefs and culture intersect. Journal of Research on Technology in Education, London, v. 42, n. 3, p. 255-284, 2010.

GIANNELLA, T. R. Inovações no ensino das ciências e da saúde: pesquisa e desenvolvimento da ferramenta Constructore e do banco virtual de neurociência. 2007. Tese (Doutorado em Química Biológica - Educação, Difusão e Gestão em Biociências) - Instituto de Bioquímica Médica, Universidade Federal do Rio de Janeiro, Rio de Janeiro, 2007.

GONÇALVES, S. M. P.; OLIVEIRA, I. R. P. Mudança na práxis: estudo de uma inovação pedagógica apoiada nas tecnologias de informação e comunicação. Eduser: revista de educação, Bragança, v. 3, n. 1, p. 1-18, 2011.

GOUDOURIS, E. S.; GIANNELLA, T. R.; STRUCHINER, M. Tecnologias de informação e comunicação e ensino semipresencial na educação médica. Revista Brasileira de

Educação Médica, Rio de Janeiro, v. 37, n. 3, p. 396-407, 2013.

GROFF, J.; MOUZA, C. A framework for addressing challenges to classroom technology use. Association for the Advancement of Computer in Education Journal, Chesapeake, v. 16, n. 1, p. 21-46, 2008.

HAGGERTY, C. Developing an online course: reflections on the process. Whitireia Nursing Journal, Wellington, NZ, v. 16, p. 8-17, 2009.

HARRIS, J.; MISHRA, P.; KOEHLER, M. Teachers' technological pedagogical content knowledge and learning activity types: curriculum-based technology integration reframed. Journal of Research on Technology in Education, JRTE, Washington, v. 41, n. 4, p. 393-416, 2009. 
Continuidade e descontinuidade de uso de tecnologias digitais ...

HU, Z; MCGRATH, I. Integrating ICT into college English: an implementation study of a national reform. Education and Information Technologies, New York, v. 17, n. 2, p. 147-165, 2012.

MCALLISTER, M. et al. Snapshots of simulation: creative strategies used by Australian educators to enhance simulation learning experiences for nursing students. Nurse Education in Practice, Edinburgh, v. 13, n. 6, p. 567-572, 2013.

MENDOZA, A.; CARROLL, J.; STERN, L. Influences on continued use of an information system: a longitudinal study. In: EUROPEAN CONFERENCE ON INFORMATION SYSTEMS, ECIS, 2008, Galway, Ireland. Proceedings...

MENEGHEL, S. M.; KREISCH, C. Concepções de avaliação e práticas avaliativas na escola: entre possibilidades e dificuldades. In: CONGRESSO NACIONAL DE EDUCAÇÃO, 9., 2009, Curitiba. Anais... Disponível em: <http://educere.bruc.com.br/arquivo/ pdf2009/3393_1920.pdf>. Acesso em: 15 ago. 2018.

MORAIS, C.; ALVES, P.; MIRANDA, L. Valorização dos ambientes virtuais de aprendizagem por professores do ensino superior. In: CONFERÊNCIA IBÉRICA DE SISTEMAS E TECNOLOGIAS DE INFORMAÇÃO E COMUNICAÇÃO, 8., 2013, Lisboa. Anais... Disponível em: <https:/ /ieeexplore.ieee.org/stamp/stamp. jsp?arnumber $=6615863>$. Acesso em: 15 ago. 2018.

MUMTAZ, S. Factors affecting teachers' use of information and communication technology: a review of the literature. Journal of Information Technology for Teacher Education, London, v. 9, n. 3, p. 319-342, 2000.

NACHMIAS, R.; RAM, J. Research insights from a decade of campus-wide implementation of web-supported academic instruction at Tel Aviv University. The International Review of Research in Open and Distance Learning, Athabasca, v. 10, n. 2, p. 1-16, 2009.

NIELSEN, J. Designing web usability: the practice of simplicity. San Francisco: New Riders Press, 1999.

OWSTON, R. Contextual factors that sustain innovative pedagogical practice using technology: an international study. Journal of Educational Change, Basel, v. 8, n. 1, p. 61-77, 2007.

PELGRUM, W. J. Obstacles to the integration of ICT in education: results from a worldwide education assessment. Computers \& Education, Amsterdam, v. 37, n. 2, p. 163-178, 2001.

RAMOS, V. Desenvolvimento de duas plataformas web integradas baseadas no conceito de objetos de aprendizagem: Constructore e banco virtual de objetos de aprendizagem. 2004. 34 f. Trabalho de conclusão de curso (Bacharelado em Ciência da Computação) - Instituto de Matemática, Universidade Federal do Rio de Janeiro, Rio de Janeiro, 2004.

RANGEL, E. et al. Avaliação, por graduandos de enfermagem, de ambiente virtual de aprendizagem para ensino de fisiologia endócrina. Acta Paulista de Enfermagem, São Paulo, v. 24, n. 3, p. 327-333, 2011. 
ROGERS, E. Diffusion of innovations.5. ed. New York: Free Press, 2003.

SILVA, I. S. A.; MARQUES, I. R. Conhecimento e barreiras na utilização dos recursos das tecnologias de informação e comunicação por docentes de enfermagem. Journal of Health Informatics, São Paulo, v. 3, n. 1, p. 3-8, 2011.

SIMÕES, R. H. S. Da avaliação da educação à educação da avaliação: o lugar do(a) educador(a) no processo da avaliação da pós-graduação no Brasil. Psicologia \& Sociedade, Belo Horizonte, v. 16, n. 1, p. 124-134, 2004. (Número especial).

SO, H.-J.; BRUSH, T. A. Student perceptions of collaborative learning, social presence and satisfaction in a blended learning environment: relationships and critical factors. Computers \& Education, Amsterdam, v. 51, n. 1, p. 318-336, 2008.

VYAS, R. et al. Clinical training at remote sites using mobile technology: an India-USA partnership. Distance Education, London, v. 31, n. 2, p. 211-216, 2010.

WARDENSKI, R. Continuidade e descontinuidade no uso de tecnologias de informação e comunicação no ensino superior: análises das percepções de professores da área da saúde com o uso da Constructore. 2016. 166 f. Dissertação (Mestrado em Educação em Ciências e Saúde) - Núcleo de Tecnologia Educacional para a Saúde, Universidade Federal do Rio de Janeiro, Rio de Janeiro, 2016.

WEINTRAUB, M.; HAWLITSCHEK, P.; JOÃO, S. M. A. Jogo educacional sobre avaliação em fisioterapia: uma nova abordagem acadêmica. Fisioterapia e Pesquisa, São Paulo, v. 18, n. 3, p. 280-286, 2011.

WEST, R. E.; WADDOUPS, G.; GRAHAM, C. R. Understanding the experiences of instructors as they adopt a course management system. Educational Technology Research and Development, Washington, v. 55, n. 1, p. 1-26, 2007.

Artigo recebido em 26/03/2018. Aceito em 11/04/2018.

Contato: Universidade Federal do Rio de Janeiro, Núcleo de Tecnologia Educacional para a Saúde, Avenida Carlos Chagas Filho, 373, Bloco L, sala 32, CEP 21941-901, Rio de Janeiro, RJ, Brasil. 